\title{
Glutamine Metabolism During Aerial Mycelium Growth of Neurospora crassa
}

\author{
By MARÍA E. CÁRDENAS AND WILHELM HANSBERG* \\ Centro de Investigación sobre Fijación de Nitrógeno, Universidad Nacional Autónoma de México, \\ Apartado Postal 565-A-Cuernavaca, Mor., Mexico
}

(Received 8 December 1983)

\begin{abstract}
During vegetative growth, glutamine is accumulated in the mycelium of Neurospora crassa. This high pool of glutamine seems to be required for aerial mycelium growth. Enzymes responsible for the synthesis and catabolism of glutamine were measured before and during the partial transformation of a mycelial mat into aerial mycelium. In the transforming mycelial mat, considerable activities of the biosynthetic NADP-glutamate dehydrogenase and glutamine synthetase (predominantly $\beta$ polypeptide) and also some activity of glutamate synthase were observed. In the aerial mycelium, glutamine synthetase (predominantly $\beta$ polypeptide) was detected, but very low activities of NADP-glutamate dehydrogenase and glutamate synthase were observed, indicating that nitrogen can be assimilated in the mycelial mat but hardly in the aerial hyphae. Accumulated glutamate in the aerial mycelium could derive from glutamine. No glutaminase activity could be detected. It is suggested that glutamate is formed through the activities of the glutamine transaminase- $\omega$-amidase pathway and another transaminase. High activities of glutamine and alanine transaminases were observed in the aerial mycelium. These results are discussed in terms of the possible role of glutamine as a nitrogen carrier from the mycelium to the growing aerial hyphae.
\end{abstract}

\section{INTRODUCTION}

When a mycelial mat of Neurospora crassa is exposed to the air in the complete absence of nutrients, it rapidly forms aerial hyphae and conidiates thereafter in a synchronous fashion (Stine \& Clark, 1967; Cárdenas \& Hansberg, 1984). After $5 \mathrm{~h}$ under these conditions, the aerial mycelium can be harvested and the mycelial mat separated in two different layers. The three fractions represent different fungal structures that are morphologically and physiologically distinct.

During aerial mycelium growth induced by starvation, the mycelial mat must supply the metabolites required to build up the aerial mycelium and conidia. Accumulated metabolites during vegetative growth could be utilized for aerial mycelium growth or some metabolites could be synthesized preferentially in the mycelium and translocated to the growing aerial mycelium. Of the five amino acids that are accumulated in the mycelium, only a high glutamine pool seems to be required for aerial mycelium growth (Cárdenas et al., 1984). In this paper we present a detailed description of the enzyme activities related to glutamine metabolism in the different fungal structures during the course of the partial transformation of a mycelial mat into an aerial mycelium.

Glutamine plays a central role in nitrogen assimilation and distribution and can also serve as a carbon and energy source. Neurospora crassa in the presence of abundant ammonium ions assimilates nitrogen via NADP-glutamate dehydrogenase and octomeric glutamine synthetase; when ammonium is limiting in the medium, nitrogen is assimilated via tetrameric glutamine synthetase and glutamate synthase (Lara et al., 1982). Glutamine synthetase is composed mainly of $\beta$ polypeptides in the first pathway and of $\alpha$ polypeptides in the second pathway (Dávila et al., 1980). 
Glutamine is very important in nitrogen distribution: gluatmine-amido transferase activities are essential for synthesis of many amino compounds. Glutamine transaminase activity could also be very important in nitrogen distribution (Espín et al., 1979). Since glutaminase could not be found in $N$. crassa under any circumstances (this paper), whenever glutamine was utilized as a nitrogen source it probably was catabolized via the glutamine transaminase- $\omega$-amidase pathway (Cooper \& Meister, 1977). To form glutamate, the resulting 2-oxoglutarate would transaminate with another amino acid. The hypothesis is discussed that accumulated glutamine in the vegetative mycelium and the actively synthesized glutamine in this structure are translocated to the growing aerial hyphae, where glutamine serves as a nitrogen source for the synthesis of the different metabolites, particularly glutamate, which is accumulated in the aerial mycelium and conidia.

\section{METHODS}

Organisms and chemicals. Stock cultures were kindly provided by J. Mora of this University. The strains used were: wild-type strain 74A, the proline auxotroph, prol-3, which grows in proline, ornithine or arginine (Vogel \& Bonner, 1954), and the glutamate auxotroph $a m-1 ;$ en $(a m)-2$, which lacks glutamate dehydrogenase enzyme and has no glutamate synthase activity (Hummelt \& Mora, 1980 b). All reagents for enzyme determinations and lactate dehydrogenase were purchased from Sigma.

Growth conditions. These were as described in the previous paper (Cardénas \& Hansberg, 1984). Additionally, the 74A strain was grown for $8 \mathrm{~h}$ on MM-sucrose without $\mathrm{NH}_{4} \mathrm{NO}_{3}$ and $5 \mathrm{mM}$-glutamine. The am-1; en $(\mathrm{am})-2$ double mutant was grown for $8 \mathrm{~h}$ in minimal medium without $\mathrm{NH}_{4} \mathrm{NO}_{3}$, supplemented with $5 \mathrm{mM}$-glucose, $25 \mathrm{mM}$-glutamine and $100 \mu \mathrm{g}$ alanine $\mathrm{ml}^{-1}$, and thereafter for $4 \mathrm{~h}$ in minimal medium without $\mathrm{NH}_{4} \mathrm{NO}_{3}$, supplemented with $25 \mathrm{~mm}$-glutamine.

Growth of aerial mycelium. Growth and separation of aerial mycelium was as described in the previous paper (Cárdenas \& Hansberg, 1984).

Enzyme assays. Mycelia were ground with dry ice and resuspended in the appropriate buffer, depending on the enzyme to be measured. Glutamine synthetase activity (EC 6.3.1.2) was assayed following the method described by Ferguson \& Sims (1971). Specific activity at $30^{\circ} \mathrm{C}$ was expressed as $\mu \mathrm{mol} \gamma$-glutamyl-hydroxamate produced $\min ^{-1}$ (mg protein $)^{-1}$. Protein was determined by the Lowry method. Glutamate dehydrogenases activities, NADP- (EC 1.4.1.4) and NAD- (EC 1.4.1.2) dependent, were measured by the procedure described by Fincham (1957). Glutamate synthase activity (EC 1 4.7.1) was assayed by the method of Boland \& Benny (1977), adapted by Hummelt \& Mora $(1980 a)$. Specific activities for the glutamate dehydrogenases and glutamate synthase at $25^{\circ} \mathrm{C}$ were expressed as $\mu$ mol oxidized $\mathrm{CoA} \mathrm{min}^{-1}(\mathrm{mg} \text { protein) })^{-1}$. Alanine transaminase activity (EC 2.6.1.2) was measured as described by Firtel \& Brackenbury (1972), except that $0.1 \mathrm{M}$-Tricine buffer was substituted by $0 \cdot 1 \mathrm{M}$-phosphate buffer. Pyruvate formation was measured in an excess of lactate dehydrogenase. Specific activity is expressed as $\mu \mathrm{mol} \mathrm{CoA}$ oxidized $\min ^{-1}(\mathrm{mg} \text { protein })^{-1}$. Activity of glutamine transaminase (EC 2.6.1.15) was determined by the disappearance of the enol-borate-phenylpyruvate complex, as described by Kupchik \& Knox (1970) but supplemented with 2 mM-pyridoxal phosphate (J. Calderón, E. Morett, \& J. Mora, unpublished). Specific activity is expressed as $\mu$ mol complex lost $\min ^{-1}(\mathrm{mg} \text { protein })^{-1}$ at $37^{\circ} \mathrm{C}$.

Oligomeric form of glutamine synthetase. Acetone powders of the different mycelia were ground with dry ice, resuspended and homogenized, at $0 \cdot 1 \mathrm{mg} \mathrm{ml}{ }^{-1}$, in buffer ( $\left.50 \mathrm{mM}^{-} \mathrm{K}_{2} \mathrm{SO}_{4}, 5 \mathrm{~mm}-\mathrm{K}_{2} \mathrm{PO}_{4}, 500 \mathrm{~mm}-\mathrm{EDTA}, \mathrm{pH} 7 \cdot 2\right)$. Samples of $0.4 \mathrm{ml}$ were layered on top of linear $5-20 \%(\mathrm{w} / \mathrm{v})$ sucrose gradients, made in the same buffer, and centrifuged at $4{ }^{\circ} \mathrm{C}$ for $12 \mathrm{~h}$ at $284000 \mathrm{~g}$ in a Beckman SW40 rotor. After centrifugation, the gradients were collected in $0.375 \mathrm{ml}$ fractions and in each fraction the glutamine synthetase activity was assayed (Limon-Lason et al., 1977).

Immunoprecipitation of glutamine synthetase. The procedure described by Palacios et al. (1977) was followed, with slight modifications. Samples of cell-free extracts containing $5 \mathrm{mg}$ protein were immunoprecipitated with $6.4 \mu \mathrm{g}$ anti-glutamine synthetase goat antibodies for $60 \mathrm{~min}$ at $4^{\circ} \mathrm{C}$. Then $40 \mu \mathrm{g}$ rabbit anti-goat $\gamma$-globulin was added to the reaction mixture, which was then centrifuged on discontinuous sucrose gradients. The immunoprecipitates were analysed by electrophoresis in SDS-urea-polyacrylamide gels (Sánchez et al., 1980). The gels were stained with $0.06 \%$ Coomassie brilliant blue in $30 \%(\mathrm{v} / \mathrm{v})$ methanol, $10 \%(\mathrm{v} / \mathrm{v})$ acetic acid solution and destained in $10 \%$ acetic acid. In order to determine the relative amount of $\alpha$ and $\beta$ polypeptides, the gels were cut and scanned at $680 \mathrm{~nm}$ in a spectrometer (Beckman, model 35).

\section{RESULTS}

The requirement of glutamine for aerial mycelium growth prompted us to analyse the nitrogen assimilation pathways during the partial transformation of a mycelial mat into an aerial 


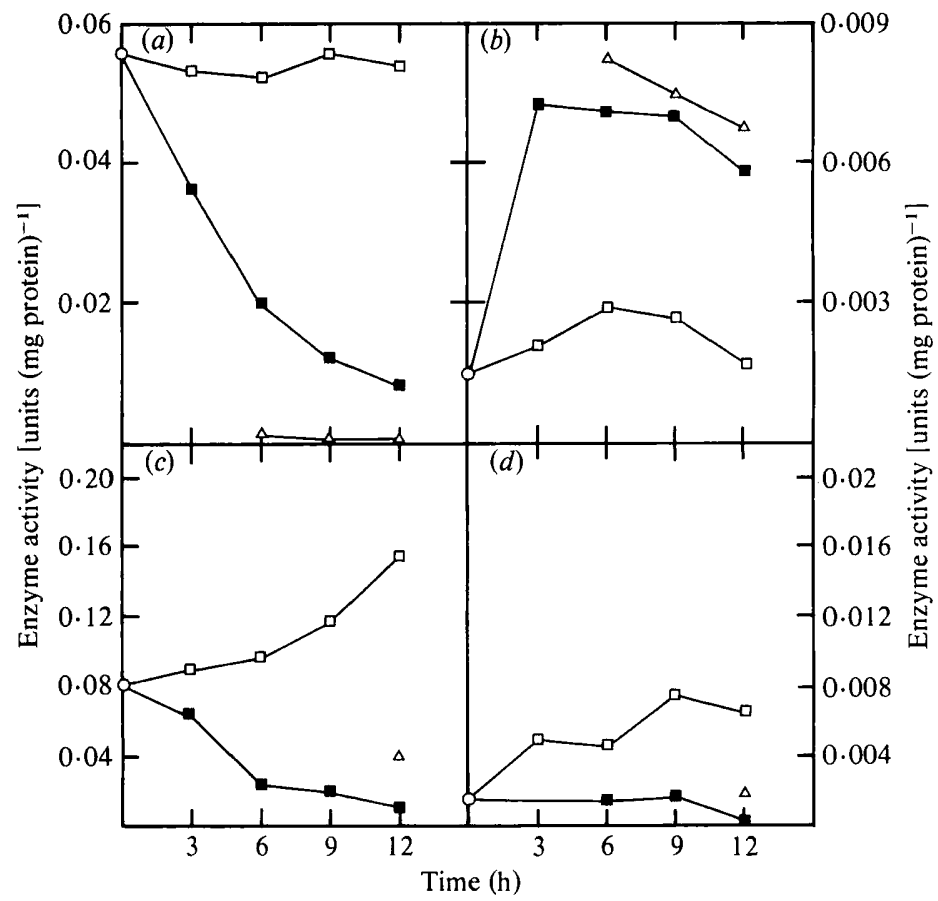

Fig. 1. Specific activities of the prol-3 NADP-glutamate dehydrogenase (a), NAD-glutamate dehydrogenase $(b)$, glutamine synthetase $(c)$ and glutamate synthase $(d)$. $\bigcirc$, Activity measured in the mycelium before starvation; $\square$, during aerial mycelium growth in the bottom layers of the mycelial mat; $\square$, in the top layer of the mycelial mat; $\Delta$, in the aerial mycelium.

mycelium. During vegetative growth, nitrogen is assimilated via the glutamate dehydrogenase (NADP)-glutamine synthetase pathway in excess ammonium, and via the glutamine synthetase-glutamate synthase pathway when ammonium is limiting (Lara et al., 1982). To determine which pathway for nitrogen assimilation is utilized in each of the different fungal structures during aerial growth induced by starvation, the activities of the glutamate dehydrogenases, glutamine synthetase, and glutamate synthase extracted from the different structures were measured at intervals during aerial mycelium growth.

High or low enzyme activities were not due to the presence in the extract of activators or inhibitors, since activities did not vary on dialysis. When extracted together, mycelia with high and low enzyme activity gave an intermediate activity.

\section{Nitrogen assimilation pathways during aerial mycelium growth}

In the lower layers of the mycelial mat, the activity of the biosynthetic, NADP-dependent glutamate dehydrogenase remained at the same level both during the whole process of aerial mycelium growth and in the mycelium at prestationary growth (Fig. 1a). Similarly, the catabolic, NAD-dependent glutamate dehydrogenase activity did not change much during the same period (Fig. $1 b$ ).

The activity of glutamine synthetase increased steadily during aerial mycelium growth and, at the end, it was about twice as high as in the mycelium at prestationary growth (Fig. $1 c$ ). The glutamate synthase activity was very low in the mycelial mat, although it increased about 4-fold during the whole process (Fig. 1d). Activity of this enzyme did not increase further when assayed after $12 \mathrm{~h}$ aerial mycelium growth (not shown). These results indicate that during prestationary growth the mycelium assimilated nitrogen by the glutamate dehydrogenaseglutamine synthetase pathway and that the activity of this pathway was preserved, or even increased, in the lower layers of the mycelial mat. The extensive protein catabolism in the 


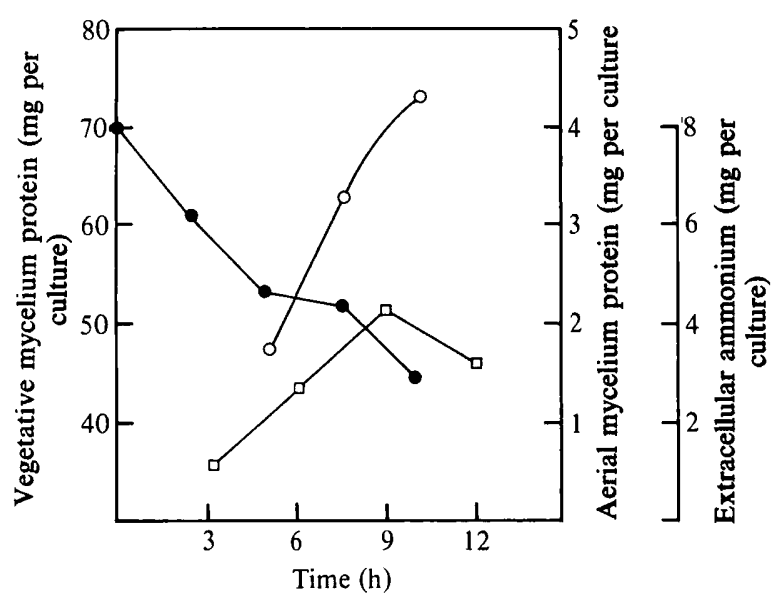

Fig. 2. Partial transformation of the mycelial mat into aerial mycelium. $\bigcirc$, Growth of aerial mycelium expressed as protein content; $\bigcirc$, protein content in the transforming mycelial mat; $\square$, extracellular ammonium. Mean values of ten, eight and two experiments, respectively.

mycelial mat (Fig. 2) could provide enough 2-oxoglutarate and ammonium to keep the nitrogen assimilation pathway active. Ammonium was produced in excess and excreted into the medium (Fig. 2).

In the uppermost layer of the mycelial mat, the activity of biosynthetic glutamate dehydrogenase decreased steadily during aerial mycelium growth and at the end of this process it was 6-7 times lower than in the mycelium during prestationary growth (Fig. 1a). The catabolic glutamate dehydrogenase in the same layer increased 3-4-fold during the first $3 \mathrm{~h}$ of aerial mycelium growth and then remained at the same level during the subsequent hours (Fig. $1 b$ ). Glutamine synthetase activity in this uppermost layer of the mycelial mat decreased steadily during aerial mycelium growth and at the end it was 7-8-fold lower than at the onset of starvation (Fig. $1 \mathrm{c}$ ). Glutamate synthase activity was almost absent in this structure during the whole process of aerial mycelium growth. In the uppermost layer of the mycelial mat there is, therefore, a considerable loss of the initial nitrogen assimilation capacity. This contrasts with the increased assimilation capacity in the lower layers of the same mycelial mat.

In the aerial mycelium, the activity of the biosynthetic glutamate dehydrogenase was almost zero (Fig. 1a). However, there was 4-5-fold more activity of the catabolic glutamate dehydrogenase than in the mycelial mat at the onset of starvation (Fig. $1 b$ ). The glutamine synthetase activity was about half of that found in the mycelium at prestationary growth and almost 4-fold lower than in the lower layers of the mycelial mat (Fig. 1c). Glutamate synthase activity in the aerial mycelium was as low as in the mycelial mat at the onset of starvation (Fig. $1 d$ ). These results indicate that in the aerial mycelium nitrogen could only be assimilated in a limited way through the glutamine synthetase activity.

\section{Oligomeric structure and polypeptide composition of glutamine synthetase}

During vegetative growth in excess ammonium, $N$. crassa glutamine synthetase has an octomeric structure which is composed mainly of $\beta$ polypeptide. However, under ammonium limitation, it is a tetramer of $\alpha$ polypeptides (Dávila et al., 1980). The $V_{\max }$ of the octomeric enzyme is 12 -fold greater than that of the tetrameric enzyme; the affinity for ammonium is 2-3fold lower (J. Guzmán \& J. Mora, unpublished).

Even though the mycelial mat was in the complete absence of external nitrogen and carbon sources, during aerial mycelium growth an increase in glutamine synthetase activity was observed in the lower layers of the mycelial mat. It was therefore of interest to determine which of the two glutamine synthetases was present during aerial mycelium growth induced by starvation. The octomeric form predominated both in the mycelium at prestationary growth and 


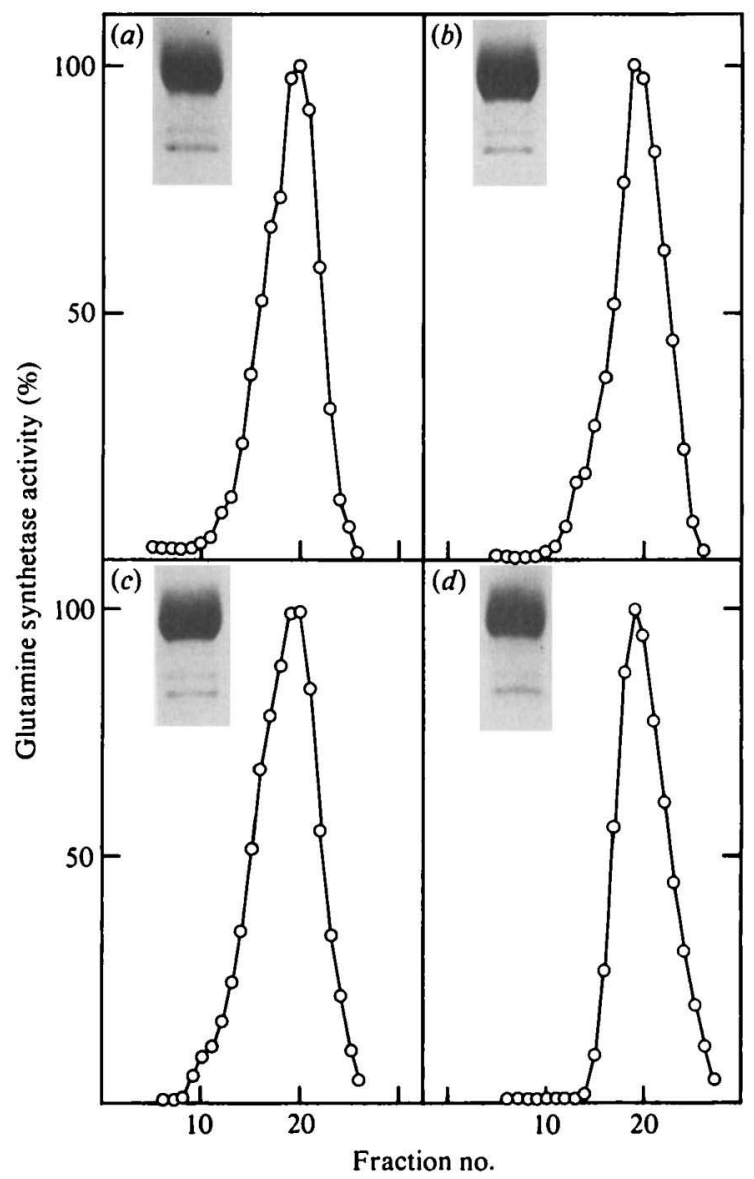

Fig. 3. Sedimentation of glutamine synthetase and polypeptide composition of glutamine synthetase. $(a)$ In the mycelium before starvation, $(b)$ in the lower layers of the mycelial mat after $12 \mathrm{~h}$ starvation, $(c)$ in the top layer of the mycelial mat at $12 \mathrm{~h}$ starvation, $(d)$ in the aerial mycelium at $12 \mathrm{~h}$. The cell-free extracts were centrifuged in 5-20\% sucrose gradients to analyse the oligomeric structure, and immunoprecipitated. The immunoprecipitate was analysed in SDS-urea polyacrylamide gels to determine the polypeptide composition.

in the three fungal structures at the end of aerial mycelium growth, and it was composed mainly of the $\beta$ polypeptides (Fig. 3). Lower oligomeric forms were also detected in the mycelium at prestationary growth and in the mycelial mat. In the aerial mycelium no lower oligomeric forms of glutamine synthetase were detected (Fig. 3d). In the lower layers of the mycelial mat the proportion of the $\alpha$ polypeptide in the immunoprecipitated glutamine synthetase increased from $27 \%$ to $44 \%$ during the first $3 \mathrm{~h}$ of aerial mycelium growth. Thereafter, the ratio of $\beta$ polypeptide to $\alpha$ polypeptide increased steadily up to a value of 2 after $12 \mathrm{~h}$ incubation (Fig. 4). A similar ratio was found in the uppermost layer of the mycelial mat at end of aerial mycelium growth, and in the aerial mycelium the ratio was 2.6 after $9 \mathrm{~h}$ growth, very similar to the ratio seen in the mycelium at prestationary growth (Fig. 4).

\section{Glutaminase and glutamine transaminase activities during aerial mycelium growth}

The conidia of $N$. crassa have very high pools of glutamate and glutamine. The accumulated glutamate is probably utilized to provide reduced coenzymes during the first hour of germination of the conidia (Schmit \& Brody, 1975). We also detected high pools of glutamate and glutamine in the aerial mycelium of different strains. The glutamate auxotroph, am-1; en(am)-2, which lacks NADP-glutamate dehydrogenase and glutamate synthase activities 


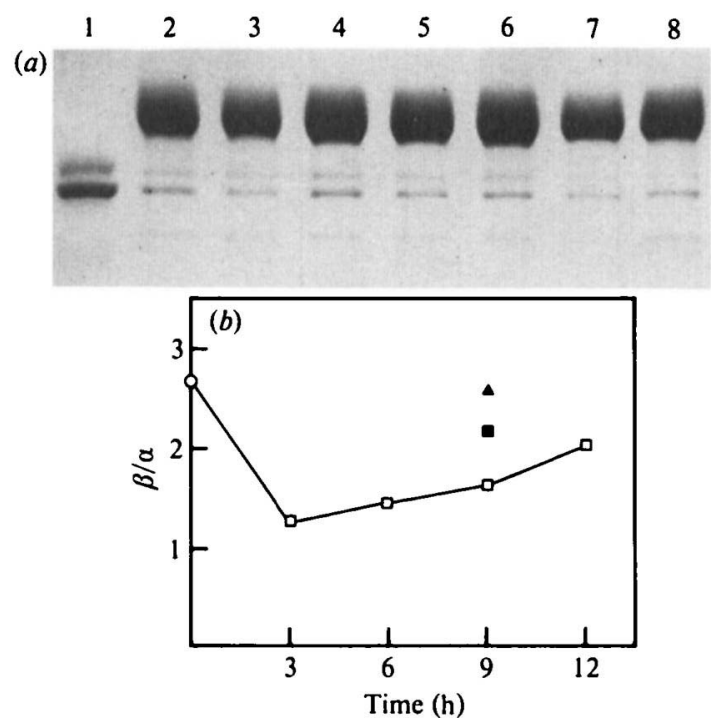

Fig. 4. Polypeptide composition of glutamine synthetase during the course of aerial mycelium growth. (a) Immunoprecipitates of glutamine synthetase: (2) in the mycelium before starvation, (3) in the lower layers of the mycelial mat after $3 \mathrm{~h},(4)$ after $6 \mathrm{~h}$, (5) after $9 \mathrm{~h}$, and (6) after $12 \mathrm{~h}$ of aerial mycelium growth; (7) in the top layer of the mycelial mat after $9 \mathrm{~h}$ starvation, and (8) in the aerial mycelium after $9 \mathrm{~h}$ growth. (1) Purified glutamine synthetase. (b) The $\beta / \alpha$ polypeptide ratio during aerial mycelium growth: $\square$, in the lower layers of the mycelial mat; $\mathbf{a}$, in the top layer; $\Delta$, in the aerial mycelium.

(Hummelt \& Mora, 1980b), also had high levels of glutamate in the aerial hyphae, even though the glutamate pool in the mycelium was depleted before the latter was starved to form aerial mycelium (Cárdenas et al., 1984). The question arises as to where the glutamate in the aerial hyphae comes from. Since accumulated glutamine in the mycelial mat was required for aerial mycelium growth, glutamine could be the source of glutamate in the aerial mycelium. Therefore the glutaminase and glutamine transaminase activities were measured in the different fungal structures during the course of aerial mycelium growth.

Glutaminase activity could not be detected under any of the different conditions assayed: various methods for enzyme determination were tried and assayed at different $\mathrm{pH}$ values in the range from 5 to 9; extracts were prepared in different ways; known activators like ATP, GTP, cAMP and $P_{i}$ were tested; and glutaminase inhibitors in the extracts were not detected. The enzyme was detected neither in extracts from mycelium of the wild type 74A strain nor in the glutamate auxotroph, am-1; en (am)-2, when they were cultured for 8 and $4 \mathrm{~h}$, respectively, in a medium with glutamine as the sole carbon and nitrogen source.

During growth of the mycelium, the glutamine transaminase activity did not vary much (Fig. $5 a$ ). After starvation under conditions of aerial mycelium growth, this enzyme activity decreased in the mycelial mat: 10 -fold in the lower layers and 2-3-fold in the uppermost layer. However, in the aerial mycelium much higher activities were found : $3-4$-fold higher than during vegetative growth and 30-40-fold higher than in the lower layers of the mycelial mat at the end of aerial mycelium growth (Fig. $5 a$ ).

\section{Possible role of alanine transaminase in the synthesis of glutamate from glutamine}

Since glutaminase could not be detected and glutamine transaminase was found to be very active in the aerial mycelium, glutamine could be catabolized by the glutamine transaminase- $\omega$ amidase pathway (Cooper \& Meister, 1977). This pathway has been found to be active in $N$. crassa (J. Calderón, E. Morett \& J. Mora, unpublished). The products of the $\omega$-amidase, 2oxoglutarate and ammonium, could not directly form glutamate in the aerial mycelium, since almost no activity of the biosynthetic glutamate dehydrogenase was detected in this structure. Glutamate has to be formed by transamination of 2-oxoglutarate and another amino acid. 


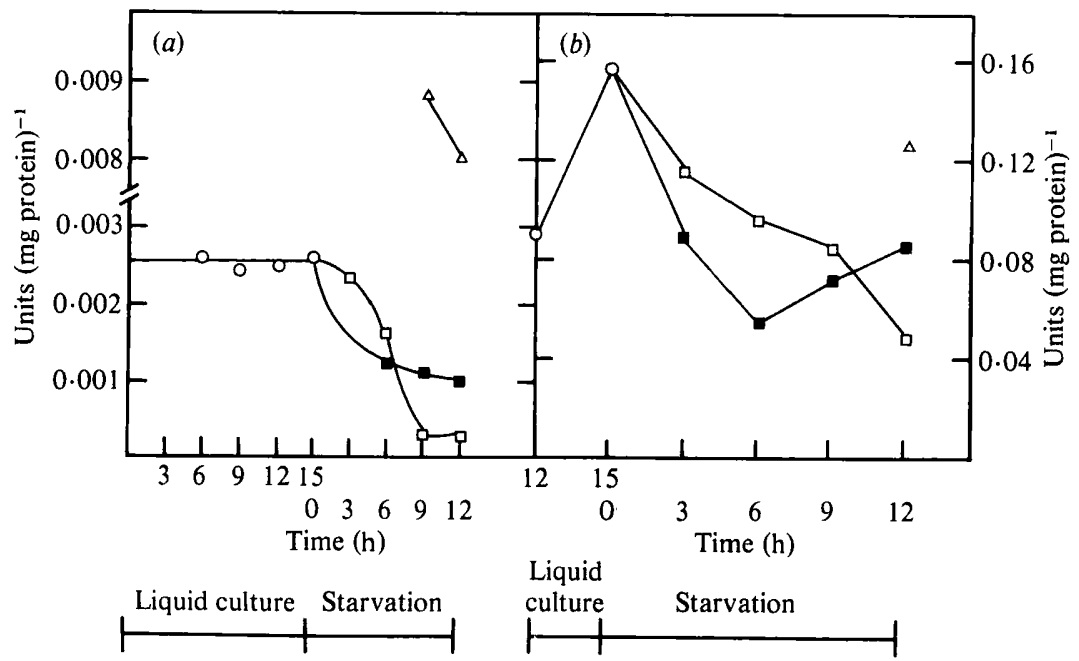

Fig. 5. Specific activities of the prol-3 glutamine transaminase $(a)$ and the alanine transaminase $(b)$. $O$, Activity measured in the mycelium before starvation; $\square$, during aerial mycelium growth in the bottom layer of the mycelial mat; $\boldsymbol{D}$, in the top layer of the mycelial mat; $\Delta$, in the aerial mycelium.

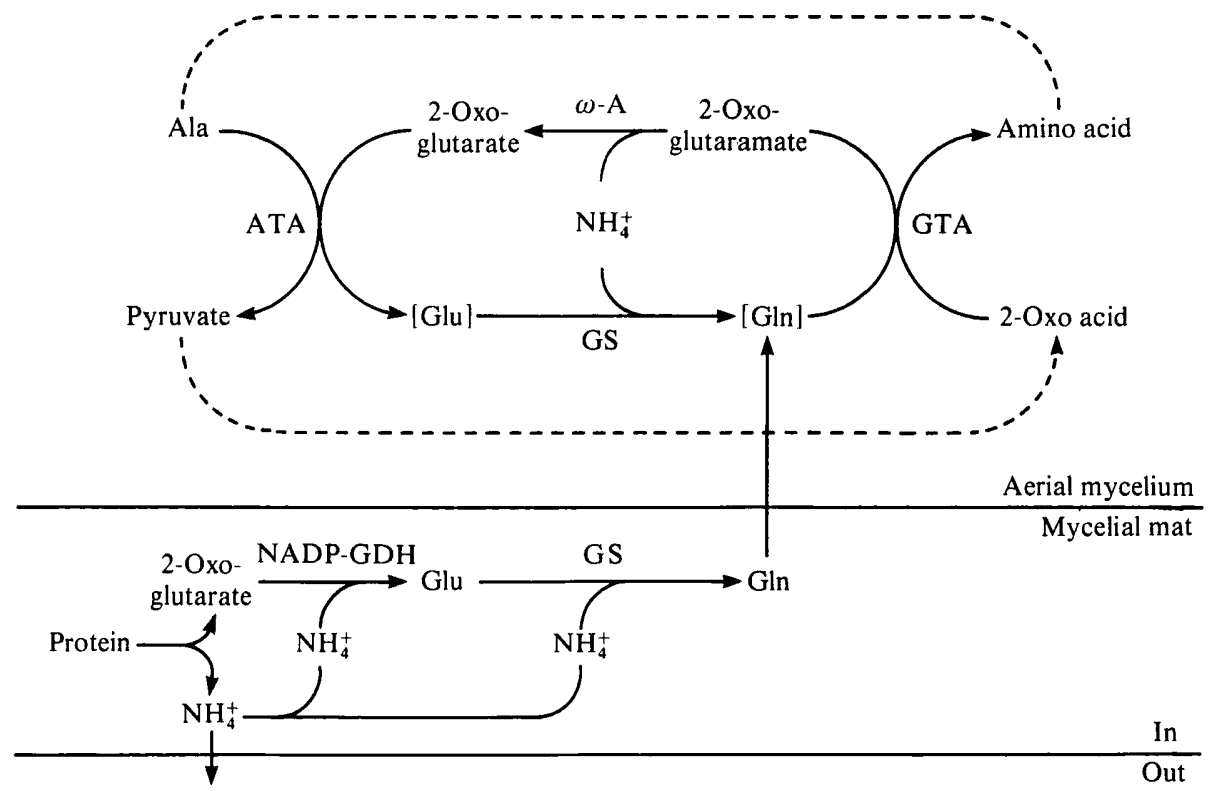

Fig. 6. Diagram of the active pathways in the different structures during aerial mycelium growth. Abbreviations: NADP-GDH, NADP-glutamate dehydrogenase; GS, glutamine synthetase; GTA, glutamine transaminase; $\omega$-A, $\omega$-amidase; ATA, alanine transaminase.

Physiological evidence has been presented to indicate that whenever glutamine was utilized as a nitrogen source, glutamate was formed by transamination of 2-oxoglutarate and alanine, particularly when the NADP-glutamate dehydrogenase activity was absent (Cárdenas et al., 1984). Alanine could be resynthesized through the glutamine transaminase activity (Fig. 6). Therefore, alanine transaminase was measured in the different fungal structures during the course of aerial mycelium growth.

The activity of alanine transaminase was very high in the mycelium during prestationary growth. When the mycelium was starved to grow aerial mycelium, this enzyme activity 
decreased markedly in the mycelial mat, 3-4-fold in the lower layers and 2-fold in the uppermost layer. However, in the aerial mycelium a high activity of alanine transaminase was detected, comparable to that found in the vegetative mycelium during prestationary growth (Fig. $5 b$ ).

\section{DISCUSSION}

During aerial mycelium growth induced by starvation, the activity of the NADP-glutamate dehydrogenase-glutamine synthetase pathway is preserved or increased in the lower layers of the mycelial mat (Fig. $1 a, c$ ). This is noteworthy, since it has been reported that in $N$. crassa this pathway is active during growth with a good carbon source and an excess of ammonium. There is a positive regulation of the carbon source on both the NADP-glutamate dehydrogenase (Kapoor \& Groves, 1970) and the glutamine synthetase (Limon-Lason et al., 1977; Mora et al., 1980). On the contrary, NAD-glutamate dehydrogenase activity increases when the carbon source is limiting (Kapoor \& Groves, 1970). The nitrogen source also exerts a similar control on these three enzymes (Hernández, 1980; Limon-Lason et al., 1977; Vichido et al., 1978). Under carbon source limitation or carbon and nitrogen limitation, the glutamine synthetase is degraded (More et al., 1980). Since (a) the NADP- and the NAD-glutamate dehydrogenases remained at the same level as during vegetative growth, $(b)$ very low levels of glutamate synthase were detected, and $(c)$ the glutamine synthetase activity increased steadily during the whole course of aerial mycelium growth and had an octomeric form composed mainly by the $\beta$ polypeptide, there is no doubt about the activity of the NADP-glutamate dehydrogenaseglutamine synthetase pathway in the lower layers of the mycelial mat. The extensive protein catabolism in the starved mycelium could provide the required 2-oxoglutarate and ammonium for this pathway (Fig. 2). Nevertheless, during the first $3 \mathrm{~h}$ of starvation a drastic fall in the $\beta / \alpha$ polypeptide ratio of the glutamine synthetase was observed. Since during this period the glutamine synthetase activity hardly changed (Fig. $1 c$ ), it has to be assumed that degradation of the $\beta$ polypeptide and synthesis of the $\alpha$ polypeptide occurs. After $3 \mathrm{~h}$ the $\beta / \alpha$ ratio and the enzyme activity increased steadily (Fig. 4 , Fig. 1c). These data indicate a transitory limitation of the nitrogen and/or the carbon source.

It is particularly noteworthy that the system active during aerial mycelium growth was the NADP-glutamate dehydrogenase-octomeric glutamine synthetase pathway, which has low substrate affinities and a high turnover rate, rather than the high-affinity, low-turnover pathway of the tetrameric glutamine synthetase-glutamate synthase (J. Guzmán \& J. Mora, unpublished). This, and the need for a high glutamine pool in the mycelial mat for aerial mycelium growth (Cárdenas \& Hansberg, 1984), indicate that there is a high requirement for glutamine for growth of the aerial mycelium. These results contrast with the low capacity for nitrogen assimilation found in the uppermost layer of the mycelial mat and in the aerial hyphae, and suggest that the accumulated and actively synthetized glutamine is probably translocated from the mycelium into the aerial mycelium, where it is utilized.

Finally, our results indicate that there is probably no activity of glutaminase in $N$. crassa. This was not surprising, since an energy-consuming cycle with glutamine synthetase could be formed. But in order to form glutamate from glutamine, as is the case when glutamine is utilized as the nitrogen source, glutamine is probably catabolized via the glutamine transaminase- $\omega$-amidase pathway (Espin et al., 1979). The resulting 2-oxoglutarate and ammonium could form glutamate with the NADP-glutamate dehydrogenase activity, but in the absence of this enzyme, 2oxoglutarate could transaminate with another amino acid to form glutamate. In the aerial mycelium, no NADP-glutamate dehydrogenase and glutamate synthase activities were found; nevertheless a high pool level of glutamate was observed. The am-1; en (am)-2 double mutant, which lacks both enzyme activities, even when deprived of glutamate, also formed aerial mycelium with a high pool level of glutamate (Cárdenas \& Hansberg, 1984). To explain these results, we propose that glutamine, which is probably translocated from the mycelium into the aerial mycelium, is catabolized in the aerial hyphae by the glutamine transaminase- $\omega$-amidase pathway and glutamate is formed by transamination of 2-oxoglutarate (Fig. 6). Since the alanine pool level decreases considerably whenever glutamine is utilized as the sole nitrogen source 
(Cárdenas \& Hansberg, 1984), alanine is probably transaminated with 2-oxoglutarate. Glutamine and alanine transaminase were very active in the aerial mycelium (Fig. $5 a, b$ ).

The physiological data of the accompanying paper and the enzymic results presented here accord with our hypothesis that glutamine serves as a nitrogen carrier from the mycelium to the growing aerial hyphae.

We are grateful to Jaime Mora for his criticism throughout this work. We also thank Emelia Breña-Valle for critically reading the manuscript, and Guadalupe Dingler for her secretarial assistance.

The research was supported in part by CONACyT (Consejo Nacional de Ciencia y Tecnología), México, grant PCCBNAL790056 to W. Hansberg. Maria E. Cárdenas was supported by an UNAM-CONACyT fellowship.

\section{REFERENCES}

Boland, M. J. \& Benny, A. G. (1977). Enzymes of nitrogen metabolism in legume nodules. Purification and properties of NADH-dependent synthase from lupin nodules. European Journal of Biochemistry 79, $355-362$.

CÁrdenas, M. E. \& Hansberg, W. (1984). Glutamine requirement for aerial mycelium growth in Neurospora crassa. Journal of General Microbiology 130, 1723-1732.

CoOper, A. J. L. \& Meister, A. (1977). The glutamine transaminase- $\omega$-amidase pathway. CRC Critical Reviews in Biochemistry 4, 281-303.

Dávila, G., Lara, M., Guzmán, J. \& Mora, J. (1980). Relation between structure and function of Neurospora crassa glutamine synthetase. Biochemical and Biophysical Research Communications 93, 134-140.

Espín, G., Palacios, R. \& Mora, J. (1979).Glutamine metabolism in conidia from Neurospora crassa. Journal of General Microbiology 115, 59-68.

Ferguson, A. R. \& Sims, A. P. (1971). Inactivation in vivo of glutamine synthetase and NAD-specific glutamate dehydrogenase: its role in the regulation of glutamine synthesis in yeasts. Journal of General Microbiology 69, 423-427.

Fincham, J. R. S. (1957). A modified glutamic acid dehydrogenase as a result of gene mutation in Neurospora crassa. Biochemical Journal 65, 721-728.

FIRTEL, R. \& BRACKenBURY, R. (1972). Partial characterization of several amino acid metabolizing enzymes in the cellular slime mold Dictyostelium discoideum. Developmental Biology 27, 307-321.

HERNÁNDEZ, G. (1980). Regulación de la deshidrogenasa glutámica biosintética de Neurospora crassa. Tesis de licenciatura, Colegio de Ciencias y Humanidades, Universidad Nacional Autónoma de México.

Hummelt, G. \& MoRA, J. (1980a). NADH-dependent glutamate synthase and nitrogen metabolism in Neurospora crassa. Biochemical and Biophysical Research Communications 92, 127-133.

Hummelt, G. \& MORA, J. (1980b). Regulation and function of glutamate synthase in Neurospora crassa. Biochemical and Biophysical Research Communications 96, 1688-1694.
KAPOOR, M. \& GROVER, A. K. (1970). Catabolitecontrolled regulation of glutamate dehydrogenases of Neurospora crassa. Canadian Journal of Microbiology 16, 33-40.

KUPCHIK, H. Z. \& KNox, W. E. (1970). Assays of glutamine and its aminotransferase with the enolborate of phenylpyruvate. Archives of Biochemistry and Biophysics 136, 178-186.

lara, M., Blanco, L., Campomanes, M., Calva, E., Palacios, R. \& MoRA, J. (1982). Physiology of ammonium assimilation in Neurospara crassa. Journal of Bacteriology 150, 105-112.

Limon-Lason, J., LARA, M., Resendiz, B. \& MoRa, J. (1977). Regulation of glutamine synthetase in fedbatch cultures of Neurospora crassa. Biochemical and Biophysical Research Communications 78, 1234 1240.

MORA, Y., ChÁvez, O. \& MORA, J. (1980). Regulation of Neurospora crassa glutamine synthetase by the carbon and nitrogen source. Journal of General Microbiology 118, 455-463.

Palacios, R., Campomanes, M. \& Quinto, C. (1977). Neurospora crassa glutamine synthetase. Translation of specific messenger ribonucleic acid in a cell free system derived from rabbit reticulocytes. Journal of Biological Chemistry 252, 3028-3034.

Sánchez, F., Calva, E., Campomanes, M., Blanco, L., Guzmán, J., Saborio, J. L. \& Palacios, R. (1980). Heterogeneity of glutamine synthetase polypeptides in Neurospora crassa. Journal of Biological Chemistry 255, 2231-2234.

SCHMIT, J. C. \& BRODY, S. (1975). Neurospora crassa conidial germination: role of endogenous amino acid pool. Journal of Bacteriology 124, 232-242.

Stine, G. J. \& Clark, A. M. (1967). Synchronous production of conidiophores and conidia of Neurospora crassa. Canadian Journal of Microbiology 13, 447453.

Vichido, I., Mora, Y., Quinto, C., Palacios, R. \& MORA, J. (1978). Nitrogen regulation of glutamine synthetase in Neurospora crassa. Journal of General Microbiology 106, 251-259. 